\title{
Stochastic Gradient Optimization Method of Mechanical Parameters of Prestressed Concrete U Shaped Girder with Gaussian Objective Function
}

\author{
Lin Su ${ }^{1, \mathrm{a}}$, Jian Zhang ${ }^{2, \mathrm{~b}}$, Cheng $\mathrm{Xi}^{3, \mathrm{c}}$, Qingyu Liang ${ }^{4, \mathrm{~d}}$ and Jiawei Tian ${ }^{2, \mathrm{e}}$ \\ 1Jiangsu Yangtze River Bridge Co., Ltd., Jiangsu Nanjing, China \\ ${ }^{2}$ Nanjing University of Aeronautics and Astronautics, Jiangsu Nanjing, China \\ ${ }^{3}$ Hohai University, Jiangsu Nanjing, China \\ ${ }^{4}$ Zhejiang Guangchuan Engineering Consulting Co., Ltd, Zhejiang Hangzhou China \\ a sulinjydq@126.com, bjianzhang78@126.com, chengxi@163.com, ${ }^{d}$ liangqy@163.com, \\ etianjiawei001@126.com
}

\begin{abstract}
Keywords: U shaped girder, mechanical parameters, Powell optimization theoretical model, Gaussian objective function.

Abstract. U shaped girder is a kind of low height bridge. The application of concrete U shaped girder is wide in highway and urban bridge. In general, it is composed of concrete and steel and compared with the mechanical analysis, little research has been carried on the inverse model of elastic modulus of U shaped girder. With degraded solid element theory, the shell element is deduced and the displacement function is obtained. Then Gaussian objective function of mechanical parameters of the reinforced concrete $U$ shaped girder is founded and the corresponding formulas of Gaussian expectation and variance are derived. The stochastic optimization estimation computing formulas are also obtained by adopting optimization method including Powell gradient method. Then the steps of stochastic Gaussian optimized estimation of mechanical parameters of the reinforced concrete U shaped girder are listed. In this paper, a reliable calculation method is provided by researching the mechanical properties for prestressed concrete continuous $U$ shaped girder.
\end{abstract}

\section{Introduction}

Prestressed concrete $U$ channel continuous rigid frame bridge is a kind of highway concrete bridge and compared with concrete box girder bridge and $\mathrm{T}$ girder bridge, it has such advantages:low height,good safety performance and low noise [1,2]. According to the characteristics of highway bridges and requirements, the new method to design and calculation method of highway concrete shaped girder is used, and not is completely copying the design and calculation method of existing railway shaped girder $[3,4]$. Concrete beam is in the form of I-shaped, $\Gamma$-shape, U-shape and box. If the span is not available, we can use the I-shaped; if not, we can use the box [5]. In order to reduce edge beam spacing, that is reducing the transverse span of driving but, concrete beam can turn into inclined edge beam, and we can use $\Gamma$-shape, $U$ shaped girder is more used in building the girders of rail transit in our country in recent years. Girder beam is beautiful in body shape and its edge beam effectively hides the deck department and vehicle [6]. Girder beam is beautiful in body shape and its edge beam effectively hides the deck department and vehicle. Especially for railway bridge and rail transit, the side beam can keep out the running system of poor appearance, and shows the upper body with neat and beautiful performance [7]. The bridge integrates into urban landscape better. The present study is aimed to implement the stochastic Gaussian optimized estimation of mechanical parameters of the reinforced concrete $U$ shaped girder [8-9]. Gaussian objective function of mechanical parameters of the reinforced concrete $U$ shaped girder is founded and the corresponding formulas are derived. The calculative procedure of optimized estimation of mechanical parameters of the reinforced concrete $U$ shaped girder is completed. 


\section{The element of the continuous $U$ shaped girder}

The continuous $U$ shaped girder consist of concrete, Common reinforced and prestressed reinforcement,the layer unit cannot accurately simulate the geometrical configuration of prestressed reinforcement, so it is simulated by the composite shell element ( as shown in Fig. 1), the node displacement array of degenerate shell element is:

$$
\delta_{i}=\left[\begin{array}{lllll}
u_{i} & v_{i} & w_{i} & \beta_{1 i} & \beta_{2 i}
\end{array}\right]^{\mathrm{T}}
$$

In this formula: $\left[\begin{array}{lll}u_{i} & v_{i} & w_{i}\end{array}\right]$ is the line displacement of node $i$ in global coordinate system; $\left[\begin{array}{ll}\beta_{1 i} & \beta_{2 i}\end{array}\right]$ is the angle displacement of node $i$ in node coordinate system.
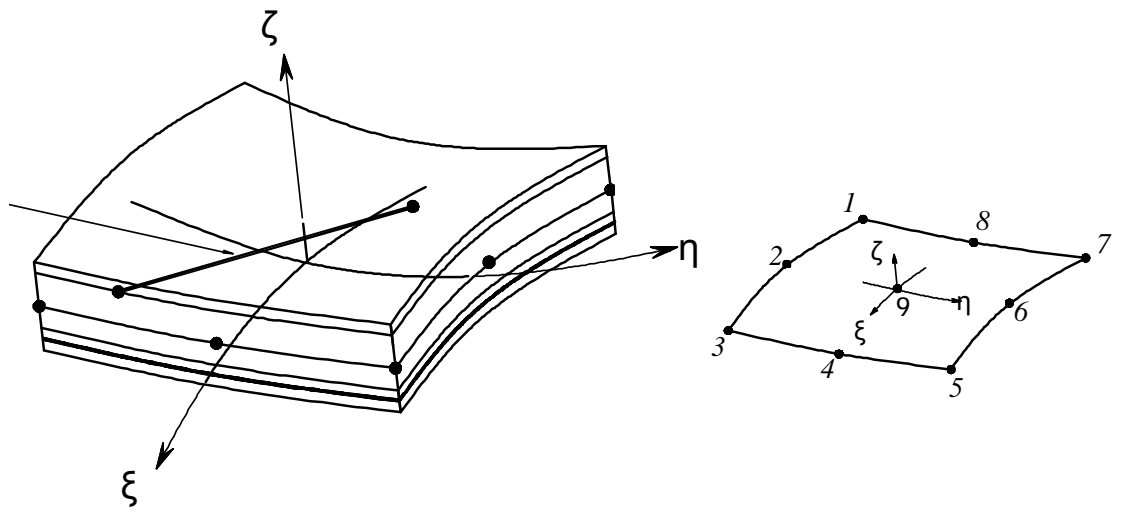

Fig. 1 Element model

Through the shape function interpolating, the displacement field shows as follows:

$$
\begin{aligned}
& u=\sum_{i=1}^{n} N_{i} u_{i}+\sum_{i=1}^{n} N_{i} \frac{h_{i}}{2} \zeta\left(v_{1 i}^{x} \beta_{1 i}-v_{2 i}^{x} \beta_{2 i}\right) \\
& v=\sum_{i=1}^{n} N_{i} v_{i}+\sum_{i=1}^{n} N_{i} \frac{h_{i}}{2} \zeta\left(v_{1 i}^{y} \beta_{1 i}-v_{2 i}^{y} \beta_{2 i}\right) \\
& w=\sum_{i=1}^{n} N_{i} w_{i}+\sum_{i=1}^{n} N_{i} \frac{h_{i}}{2} \zeta\left(v_{1 i}^{z} \beta_{1 i}-v_{2 i}^{z} \beta_{2 i}\right)
\end{aligned}
$$

In this formula: $u 、 v 、 w$ is the displacement field of degenerate shell element; $n$ is the sum of node about degenerate shell element; $N_{i}$ is the shape function of node I; $h_{i}$ is the shell element thickness of node $\mathrm{I} ; v_{1 i}^{x}$ is the arccosine of node coordinate system $v_{1}$ to the $\mathrm{x}$ axis in the global coordinate system,so is $v_{1 i}^{y}$ to $\mathrm{y}$ axis and $v_{1 i}^{z}$ to $\mathrm{z}$ axis; $v_{2 i}^{x}$ is the arccosine of node coordinate system $v_{2}$ to the $\mathrm{x}$ axis in the global coordinate system, so is $v_{2 i}^{y}$ to $\mathrm{y}$ axis and $v_{2 i}^{z}$ to $\mathrm{z}$ axis.

$$
\begin{aligned}
& N_{i}=\int_{-h / 2}^{h / 2} \sigma_{i} d z=\frac{h}{2} \sum_{m=1}^{n} \sigma_{i}^{m} \Delta \zeta^{m} \\
& M_{i}=-\int_{-h / 2}^{h / 2} \sigma_{i} z d z=-\frac{h^{2}}{4} \sum_{m=1}^{n} \sigma_{i}^{m} \zeta^{m} \Delta \zeta^{m} \\
& Q_{i}=\int_{-h / 2}^{h / 2} \tau_{i z} d z=\frac{h}{2} \sum_{m=1}^{n} \tau_{i z}^{m} \Delta \zeta^{m}
\end{aligned}
$$

Where $N_{i}, M_{i}$ and $Q_{i}$ are respectively the axial force, bending moment and shearing force. $\sigma_{i}$ is the normal stress component. $\tau_{i z}$ is the shearing stress component along the global coordinate $z$ direction. $i$ is the orientation index, $i=1$ means the global coordinate $x$ direction and $i=2$ means the $y$ direction. $n$ and $h$ are respectively the number of layers and the thickness of the element. The stiffness matrix of the layered shell element is tackled as 


$$
\mathbf{K}^{e}=\sum_{m=1}^{n} \mathbf{K}_{m}^{e}=\sum_{m=1}^{n} \int_{-1}^{1} \int_{-1}^{1} \int_{-1}^{1} \mathbf{B}_{m}^{T}(\xi, \eta, \zeta) \mathbf{D}_{m} \mathbf{B}_{m}(\xi, \eta, \zeta) d \xi d \eta d \zeta
$$

Where $\mathbf{K}^{e}$ is the stiffness matrix of the layered shell element. $\mathbf{K}_{m}^{e}$ is the stiffness matrix of the $m$ th layer, which is finally carried out by Gaussian integral method. $\mathbf{B}_{m}$ and $\mathbf{D}_{m}$ are respectively the strain matrix and the elastic matrix of the $m$ th layer of the layered shell element.

\section{Gaussian Objective Function}

Mechanical parameters of the reinforced concrete U shaped girder include Young's modulus, Poisson's ratio and others, among which Young's modulus is the most important and has great effect on the structural mechanical responses. Young's modulus is treated as estimation parameter here for simplicity. During the process of stochastic optimized estimation of mechanical parameters of the reinforced concrete U shaped girder, Young's modulus of the top and abdomen plates are both viewed as optimized estimation variables in order to carry on the poly-parameters optimized estimation. It is supposed that mechanical parameters $\mathbf{E}$ are fitted to Gaussian distribution and both means and variances of $\mathbf{E}$ are known. That is, the pre-testing distribution $f(\mathbf{E})$ is known. Bayesian parametric back analysis estimation is a theory that when the conditional distribution of the measuring displacements $f\left(\mathbf{U}^{*} \mid \mathbf{E}\right)$ and the pre-testing distribution $f(\mathbf{E})$ are given, the statistical properties of mechanical parameters are the needed quantity only if the post-testing distribution $f\left(\mathbf{E} \mid \mathbf{U}^{*}\right)$ gets its maximum. From Bayesian theory, $f\left(\mathbf{E} \mid \mathbf{U}^{*}\right)$ is

$$
f\left(\mathbf{E} \mid \mathbf{U}^{*}\right)=\frac{f\left(\mathbf{U}^{*} \mid \mathbf{E}\right) f(\mathbf{E})}{f\left(\mathbf{U}^{*}\right)}
$$

If the pre-known information about the parameters $\mathbf{E}$ can not be held accurately, the Gaussian objective function $J_{G}$ can be diverted into the following form

$$
J_{G}=\sum_{i=1}^{n}\left(\mathbf{U}_{i}^{*}-\mathbf{U}_{i}\right)^{T} \mathbf{C}_{\mathbf{U}_{i}^{*}}^{-1}\left(\mathbf{U}_{i}^{*}-\mathbf{U}_{i}\right)
$$

Where $J_{G}$ is the Gaussian objective function of the reinforced concrete $U$ shaped girder. The partial differentiation of dynamic objective function $J_{G}$ to mechanical parameters $\mathbf{E}$ is

$$
\nabla J_{G}=\sum_{i=1}^{n} 2\left(\frac{\partial \mathbf{U}_{i}}{\partial \mathbf{E}}\right)^{T} \mathbf{C}_{\mathbf{U}_{i}^{*}}^{-1}\left(\mathbf{U}_{i}-\mathbf{U}_{i}^{*}\right)
$$

\section{Steps of Stochastic Gaussian Optimization Estimation}

The Powell method is an effective method in the existing direct optimization methods and it uses one-dimensional search method to produce the optimal orientations from different starting search points.

Combined with Powell theory, the flowchart of the updating Bayesian detection of mechanical constants of the thin walled box girder is shown in Fig.2 and the detection steps are expressed as follows:

(1)Select the initial values $\boldsymbol{E}^{0,0}$ of mechanical constants $\boldsymbol{E}$ and the initial search direction $\boldsymbol{d}^{0, i}$ and let $\boldsymbol{d}^{0, i}=\boldsymbol{e}_{i}$, where $\boldsymbol{e}_{i}$ is the unit coordinate vector and $m$ is the dimension of mechanical constants $\boldsymbol{E}$ and the variable $i=1,2, \mathrm{~L}, m$. Set the iterative variable $k=0$ and convergence criteria $\varepsilon_{1}$ and $\varepsilon_{2}$; (2)With mechanical constants $\boldsymbol{E}^{k, 0}$, carry out one-dimensional search in turn along the search direction $\boldsymbol{d}^{k, i}$ where $i=1,2, \mathrm{~L}, m$, which means that:

$$
J\left(\boldsymbol{E}^{k, i}\right)=\min _{\lambda} J\left(\boldsymbol{E}^{k, i-1}+\lambda \boldsymbol{d}^{k, i}\right)
$$


And then the displacement constant series $\boldsymbol{E}^{k, i}$ are obtained. From objective function Eq.(10), we have:

$$
\Delta_{l}^{k}=\max _{1 \leq i \leq m} \Delta_{i}^{k}=\max _{1 \leq i \leq m}\left[J\left(\boldsymbol{E}^{k, i-1}\right)-J\left(\boldsymbol{E}^{k, i}\right)\right]
$$

(3)Begin with mechanical constants $\boldsymbol{E}^{k, m}$ and take one-dimensional search into action along the search direction $\boldsymbol{d}^{k}=\boldsymbol{E}^{k, m}-\boldsymbol{E}^{k, 0}$, which means that:

$$
J\left(\boldsymbol{E}^{k+1,0}\right)=\min _{\lambda} J\left(\boldsymbol{E}^{k, m}+\lambda \boldsymbol{d}^{k}\right)
$$

And then the mechanical constants $\boldsymbol{E}^{k+1,0}$ are gained. If one of the two convergence criteria can be satisfied, it is that:

$$
\begin{aligned}
& \left|\frac{J\left(\boldsymbol{E}^{k+1,0}\right)-J\left(\boldsymbol{E}^{k, 0}\right)}{J\left(\boldsymbol{E}^{k, 0}\right)}\right|<\varepsilon_{1} \\
& \left\|\frac{\boldsymbol{E}^{k+1,0}-\boldsymbol{E}^{k, 0}}{\boldsymbol{E}^{k+1,0}}\right\|_{2}<\varepsilon_{2}
\end{aligned}
$$

Then Powell iteration is convergent and the detection results of mechanical constants $\boldsymbol{E}$ are $\hat{\boldsymbol{E}}=\boldsymbol{E}^{k+1,0}$. The iteration is stopped. Otherwise, continue the next step;

(4)This step is the judgment computation whether the search direction $\boldsymbol{d}^{k}$ is collected. Assumed that $\boldsymbol{E}^{k, 2 m}=2 \boldsymbol{E}^{k, m}-\boldsymbol{E}^{k, 0}$, the following equations are gained:

$$
\begin{aligned}
J_{1}^{k} & =J\left(\boldsymbol{E}^{k, 0}\right) \\
J_{2}^{k} & =J\left(\boldsymbol{E}^{k, m}\right) \\
J_{3}^{k} & =J\left(\boldsymbol{E}^{k, 2 m}\right) \\
J_{4}^{k} & =\left(J_{1}^{k}-2 J_{2}^{k}+J_{3}^{k}\right)\left(J_{1}^{k}-J_{2}^{k}-\Delta_{l}^{k}\right)^{2} \\
J_{5}^{k} & =\frac{1}{2} \Delta_{l}^{k}\left(J_{1}^{k}-J_{3}^{k}\right)^{2}
\end{aligned}
$$

If $J_{3}^{k} \geq J_{1}^{k}$, it is useless or helpless to collect the search direction $\boldsymbol{d}^{k}$. Thus, keep the available search direction and turn to step (6). Otherwise, proceed to the next step;

(5)If $J_{4}^{k} \geq J_{5}^{k}$, the available search direction set is unchanged and proceed to step (6). Otherwise, the calculation called collecting the search direction $\boldsymbol{d}^{k}$ is finished, in which the search direction $\boldsymbol{d}^{k, l}$ in the available search directions is ignored and the search direction $\boldsymbol{d}^{k}$ is collected to become the $m$ th search direction:

$$
\begin{aligned}
& \boldsymbol{d}^{k+1, i}=\boldsymbol{d}^{k, i},(i=1,2, \mathrm{~L}, l-1) \\
& \boldsymbol{d}^{k+1, i}=\boldsymbol{d}^{k, i+1}(i=l, l+1, \mathrm{~L}, m-1) \\
& \boldsymbol{d}^{k+1, m}=\boldsymbol{d}^{k}
\end{aligned}
$$

Let $\boldsymbol{E}^{k, 0}=\boldsymbol{E}^{k+1,0}, \boldsymbol{d}^{k, i}=\boldsymbol{d}^{k+1, i}, k=k+1$ and turn back to step (2) to keep iterating;

(6)Let $\boldsymbol{E}^{k, 0}=\boldsymbol{E}^{k+1,0}, k=k+1$ and turn back to step (2) to keep iterating.

\section{Depiction of the U Shaped girder}

The width of the continuous $U$ shaped girder is $1 \mathrm{~m}$, and the combination span is $2 \times 30 \mathrm{~m}$. The high of girder beams is $1.8 \mathrm{~m}$, the width is $0.75 \mathrm{~m}$, and the bottom width is $0.35 \mathrm{~m}$; The thick in the edge of carriageway plate is $0.25 \mathrm{~m}$, the thick in the middle is $0.3 \mathrm{~m}$; The width of end cross beam is $1.0 \mathrm{~m}$ and it must reserve enough expansion joint. The width in middle beam is $1.2 \mathrm{~m}$, A cross beam across is setted every $4 \mathrm{~m}$ in the beam, the width of the cross beam is $0.4 \mathrm{~m}$, s shown in Fig. 3. Design vehicle load: highway load II. The width of the bridge deck is $6.0 \mathrm{~m}, 0.75 \mathrm{~m}$ (main beam) $+4.5 \mathrm{~m}$ (carriageway plate) $+0.75 \mathrm{~m}$ (main beam), cast-in-place concrete is made up of C50, the pier is made up C30, prestressed steel has a low relaxation and high strength steel prestressed,It is $\varphi s 15.2$ meeting GB/T 5224-2003, its nominal diameter is $\Phi s=15.2 \mathrm{~mm}$, and its nominalarea is $A=140 \mathrm{~mm} 2$, its axial tensile 
strength is $1860 \mathrm{MPa}$, its elastic modulus is $\mathrm{Ep}=1.95 \times 105 \mathrm{MPa}$, The relaxation rate is not greater than 3.5. Ordinary reinforced uses R 235 and HRB 335 reinforced,The bearing uses basin rubber type. The elastic modulus of the bottom board is written as $E_{b}$ and that of the side beam is $E_{s}$. The elastic modulus vector $\mathbf{E}$ are $\left[E_{b}, E_{s}\right]$, which are both equal to $40 \mathrm{GPa}$. In order to carry on Powell optimization theoretical inverse model of elastic modulus, the corresponding filtering procedure is developed. The $\mathrm{U}$ shaped girder is modeled by layered shell element. Using composite shell element model and considering material nonlinearity, the finite element model as shown in Fig. 4:
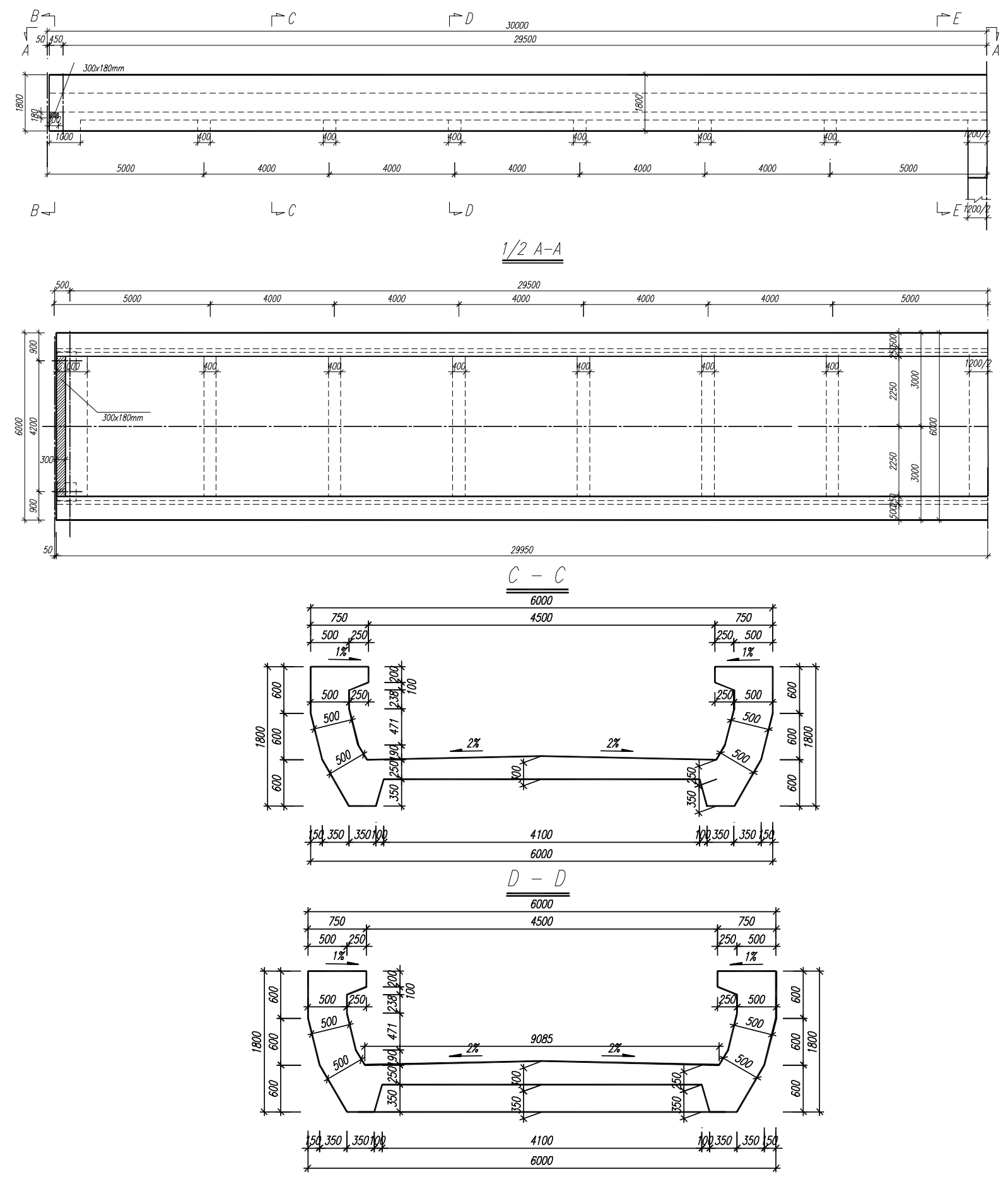

Fig. 2 General construction figure of the continuous U shaped girder(unit:mm) 

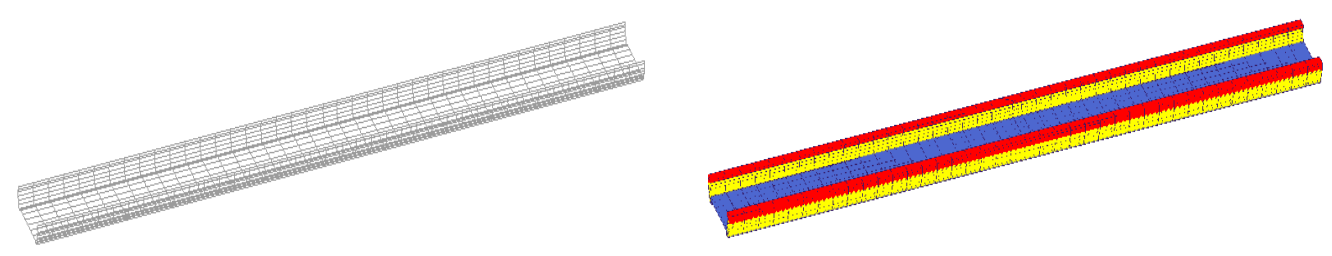

Fig. 3 finite element model of the continuous U shaped girder

\section{Analysis of Stochastic Gaussian Optimization Computation of Mechanical Parameters of the U Shaped girder}

As stated in the above, the reinforced concrete $U$ shaped girder is divided into two parts named the bottom board and the side board. The two equivalent concrete modulus are written down. Respectively select the initial values $\boldsymbol{E}_{1}=[80,80]$ and $\boldsymbol{E}_{2}=[15,15]$, which are put into the optimization analysis procedure together with the data in Table 1. The unit of the data is GPa. Suppose the convergence criteria $\varepsilon=0.1$ and the iterative results of mechanical parameters are gained in Table 1 .

Table 1 Results of stochastic optimization computation of the U shaped girder /GPa

\begin{tabular}{ccccc}
\hline Mechanical & $E_{b}$ & $E_{b}$ & $E_{s}$ & $E_{s}$ \\
Parameters & 80 & 80 & 15 & 15 \\
\hline Initial value & 40.426 & 40.157 & 39.873 & 39.558 \\
Final value & 24 & 24 & 28 & 28 \\
Iterative times & & &
\end{tabular}

From Table 1, It is indicated that when the initial values $\boldsymbol{E}_{1}=[80,80]$, the optimization results of the mechanical parameters are $[40.426,40.157]$. And when the initial values $\boldsymbol{E}_{2}=[15,15]$, those of the mechanical parameters are $[39.873,39.558]$. Evidently, differences between the two groups of results are very small. During iterative process, Gaussian estimation function can consider displacement measuring data of different times at the same time, which is efficient in computation. The iterative process of stochastic optimization computation of mechanical parameters of the prestressed concrete $\mathrm{U}$ shaped girder is steady and when different initial parameter values are set. The Powell optimization method can be used in other kinds of research work.

\section{Conclusion}

Stochastic gradient optimization computation of mechanical parameters of the prestressed concrete $\mathrm{U}$ shaped girder is studied in this paper. From theoretic research and analysis of examples, some conclusions are drawn. The iterative process of stochastic gradient optimization computation of mechanical parameters of the prestressed concrete $U$ shaped girder is steadily convergent to the same values and the convergence property of mechanical parameters is not affected by the number of random variables. Gaussian objective function of mechanical parameters of the prestressed concrete U shaped girder considers the measuring displacement data altogether, which proves the simplicity and practicability of the gradient optimization method.

\section{Acknowledgments}

This project was finally supported by National Natural Science Foundation of China(No. 11232007), Natural Science Foundation of Jiangsu Province(No. BK20130787), the Fundamental Research Funds for the Central Universities (No. NS2014003), Research Fund of Zhejiang Guangchuan Engineering Consulting Co., Ltd(No. Y1704), Research Fund of Graduate Education and Teaching Reform of NUAA(No. 2017-2), Research Fund of Education and Teaching Reform of College of Aerospace Engineering, NUAA(No. 2017-5). 


\section{References}

[1] J. Zhang, C.W. Zhou, W.G. Lan, Nonlinear dynamical identification of displacement parameters of multi-cell curve box based on Gaussian error theory, Chinese J. Applied Mech., 27 (2010), 746-750.

[2] M. S. Khaled, B. K. John, Literature review in analysis of box-girder bridges, J. Bridge Eng., 7 (2002), 134-143.

[3] J. Zhang, C.W. Zhou, X. Lei , Nonlinear beam-shell composite element of HPC beam mixed with CFRP/GFRP rebars, Acta Mater. Comp. Sin., 27 (2010), 139-146.

[4] K. Babu, M. Devdas, Correction of errors in simplified transverse bending analysis of concrete box-girder bridges, J. Bridge Eng., 10 (2005), 650-657.

[5] X.J. Wei, J.J. Zhang, S.M. Zhang, Group for shield tunnel construction induced ground maximal settlement, Rock Soil Mech., 29 (2008) 445-448.

[6] J. Zhang, J.S. Ye, C.Q. Wang, Dynamic Bayesian estimation of displacement parameters of continuous thin-walled straight box with segregating slab based on CG method, Chinese J. Comp. Mech., 25 (2008), 574-580.

[7] R.A. Ghani, U. Hangang, Thin-walled multicell straight box-girder finite element, J. Struct. Eng., 117 (1991), 2953-2971.

[8] J. Zhang, J.S. Ye, C.W. Zhou, Powell's optimal identification of material constants of thin-walled box girders based on Fibonacci series search method, Applied Math. Mech., 32 (2011), 97-106.

[9] Q.Z. Luo, Q.S. Li, J. Tang, Shear lag in box girder bridges, J. Bridge Eng., 7 (2002), 308-313. 